\title{
Review and Study of Some Doubly-Weighted Pseudo-almost Periodic Solutions of Some Partial Evolution Equations
}

\author{
BAAZAOUI Riadh* \\ Department of Mathematics, Faculty of Sciences of Tunis, University Tunis El-Manar, \\ 1060 Tunis, Tunisia.
}

Received 7 October 2015; Accepted 5 February 2017

\begin{abstract}
In this work, a characterization of the doubly-weighted Bohr spectrum of an almost periodic function is given. We showed precisely that such a spectrum is either empty or coincides with the Bohr spectrum of that function. Next, we investigate the existence of doubly-weighted pseudo-almost periodic solutions to some classes of non-autonomous partial evolution equations.
\end{abstract}

AMS Subject Classifications: 34G20, 34B05, 42A75, 47D06, 35L90

Chinese Library Classifications: $\mathrm{O} 175.26$

Key Words: Evolution family; exponential dichotomy; acquistapace and Terreni conditions; doublyweighted pseudo-almost periodic; evolution equation; nonautonomous equation.

\section{Introduction}

In [1], results on the existence and uniqueness are provided for doubly-weighted pseudoalmost periodic solution for equations

$$
u^{\prime}(t)=A(t) u(t)+g(t, u(t)), \quad t \in \mathbb{R},
$$

where $A(t)$ for $t \in \mathbb{R}$ is a family of closed linear operators on $D(A(t))$ satisfying the wellknown Acquistapace-Terreni conditions, and $g: \mathbb{R} \times \mathbb{X} \rightarrow \mathbb{X}$ is doubly-weighted pseudoalmost periodic in $t \in \mathbb{R}$ uniformly in the second variable.

The existence of weighted pseudo-almost periodic, weighted pseudo-almost automorphic, and pseudo-almost periodic solutions to differential equations constitutes one

${ }^{*}$ Corresponding author. Email address: riadh. baazaoui@fst.rnu.tn (R. Baazaoui) 
of the most attractive topics in qualitative theory of differential equations due to possible applications. Some contributions on weighted pseudo-almost periodic functions, their extensions, and their applications to differential equations have recently been made, among them are for instance [2-17] and the references therein. However, the problem which consists of the existence of doubly-weighted pseudo-almost periodic(mild) solutions to evolution equations in the form (1.1) is quite new and untreated and thus constitutes one of the main motivations of the theme. In the present work, we consider a more general setting and use slightly different techniques to study the existence of doublyweighted pseudo-almost periodic solutions to the following classes of partial evolution equations

$$
\frac{\mathrm{d}}{\mathrm{d} t}[u(t)+f(t, B(t) u(t))]=A(t) u(t)+g(t, C(t) u(t)), \quad t \in \mathbb{R},
$$

where $A(t)$ for $t \in \mathbb{R}$ is a family of closed linear operators on $D(A(t))$ satisfying the well-known Acquistapace-Terreni conditions, $B(t), C(t)(t \in \mathbb{R})$ are families of (possibly unbounded) linear operators, and $f: \mathbb{R} \times \mathbb{X} \mapsto \mathbb{X}_{\beta^{\prime}}^{t} g: \mathbb{R} \times \mathbb{X} \mapsto \mathbb{X}$ are doubly-weighted $(\mu, v)$-pseudo almost periodic in $t \in \mathbb{R}$ uniformly in the second variable.

The paper is organized as follows: Section 2 is devoted to preliminary results related to the existence of an evolution family, intermediate spaces, properties of weights, and basic definitions and results on the concept of doubly-weighted pseudo-almost periodic functions. In Section 3 we study the existence of a doubly-weighted mean for almost periodic functions. In Section 4 we prove the existence of doubly-weighted pseudo-almost periodic solutions to (1.2). In Section 5, we give an application to illustrate our results.

\section{Preliminaries}

\subsection{Evolution family and exponential dichotomy}

In this section, we introduce the inter and extrapolation spaces for $A(t)$. Consider a family of closed linear operators $A(t)$ for $t \in \mathbb{R}$ on $\mathbb{X}$ with domain $D(A(t))$ satisfying the so-called Acquistapace-Terreni conditions:

$H_{0}: \quad \exists \omega \in \mathbb{R}, \theta \in\left(\frac{\pi}{2}, \pi\right), K, L \geq 0$ and $\mu, v \in(0,1]$ with $\mu+v>1$ such that

$$
\begin{aligned}
& \Sigma_{\theta} \cup\{0\} \subset \rho(A(t)-\omega) \ni \lambda,\|R(\lambda, A(t)-\omega)\| \leq \frac{K}{1+|\lambda|}, \\
& \|(A(t)-\omega) R(\lambda, A(t)-\omega)[R(\omega, A(t))-R(\omega, A(s))]\| \leq L \frac{|t-s|^{\mu}}{|\lambda|^{v}}
\end{aligned}
$$

for $t, s \in \mathbb{R}, \lambda \in \Sigma_{\theta}:=\{\lambda \in \mathbb{C} \backslash\{0\}:|\arg \lambda| \leq \theta\}$.

For a given family of linear operators $A(t)$, the existence of an evolution family associated with it is not always guaranteed. However, if $A(t)$ satisfies Acquistapace-Terreni, 
then there exists a unique evolution family (see [18-20])

$$
\mathcal{U}=\{U(t, s): t, s \in \mathbb{R} \text { such that } t \geq s\}
$$

on $\mathbb{X}$ associated with $A(t)$.

Definition 2.1. An evolution family $(U(t, s))_{t \geq s}$ on a Banach space $\mathbb{X}$ is called hyperbolic (or has exponential dichotomy) if there exist projections $P(t), t \in \mathbb{R}$, uniformly bounded and strongly continuous in $t$, and constant $N>1, \delta>0$ such that

- (i) $U(t, s) P(s)=P(t) U(t, s)$ for $t \geq s$ and $t, s \in \mathbb{R}$;

- (ii) the restriction $U_{Q}(t, s): Q(s) \mathbb{X} \rightarrow Q(t) \mathbb{X}$ of $U(t, s)$ is invertible for $t \geq s$ and $t, s \in \mathbb{R}$ (and we set $\left.U_{Q}(t, s)=U(s, t)^{-1}\right)$;

- (iii)

$$
\|U(t, s) P(s)\| \leq N e^{-\delta(t-s)} \quad \text { and } \quad\left\|U_{Q}(s, t) Q(t)\right\| \leq N e^{-\delta(t-s)}, t \geq s, t, s \in \mathbb{R} .
$$

Here and below we set $Q:=I-P$.

Next, in the sequel we make extensive use of the real interpolation spaces of order $(\alpha, \infty)$ between $\mathbb{X}$ and $D(A(t))$, where $\alpha \in(0,1)$. See [21-23] for proofs and further information on theses interpolation spaces.

Let $A$ be a sectorial operator on $\mathbb{X}$ and $\alpha \in(0,1)$. The real interpolation space is

$$
\mathbb{X}_{\alpha}^{A}=\left\{x \in \mathbb{X},\|x\|_{\alpha}^{A}:=\sup _{r>0}\left\|r^{\alpha}(A-\omega) R(r, A-\omega) x\right\|<\infty\right\} .
$$

It is straightforward that

$$
\mathbb{X}_{0}^{A}:=\mathbb{X},\|x\|_{0}^{A}:=\|x\|, \mathbb{X}_{1}^{A}:=D(A),
$$

and $\|x\|_{1}^{A}:=\|(\omega-A) x\|$. Moreover, let $\hat{\mathbb{X}}^{A}:=\overline{D(A)}$ of $\mathbb{X}$. In particular, we will frequently be using the following continuous embedding

$$
D(A) \hookrightarrow \mathbb{X}_{\beta}^{A} \hookrightarrow D\left((\omega-A)^{\alpha}\right) \hookrightarrow \mathbb{X}_{\alpha}^{A} \hookrightarrow \hat{\mathbb{X}}^{A} \subset \mathbb{X},
$$

for all $0<\alpha<\beta<1$, where the fractional powers are defined in the usual way. We also use the following continuous embedding.

$$
\mathbb{X}_{\beta}^{A} \hookrightarrow \overline{D(A)}\|\cdot\|_{\alpha}^{A},
$$

for $0<\alpha<\beta<1$.

Given the family of linear operators $A(t)$ for $t \in \mathbb{R}$, satisfying (H1), we set

$$
\mathbb{X}_{\alpha}^{t}:=\mathbb{X}_{\alpha}^{A(t)}, \quad \widehat{\mathbb{X}}^{t}:=\hat{\mathbb{X}}^{A(t)},
$$


for $0 \leq \alpha \leq 1$ and $t \in \mathbb{R}$, with the corresponding norms.

Next, for $t \in \mathbb{R}$, we set $\mathbb{X}_{\alpha}^{t}:=\mathbb{X}_{\alpha}^{A(t)}$ and $\widehat{\mathbb{X}}^{t}:=\widehat{\mathbb{X}}^{A(t)}$. It holds that the evolution family $(U(t, s))_{t \geq s}$ generated by $A(t)$ has an exponential dichotomy with constants $N>0, \delta>$ 0 , and dichotomy projections $P(t), t \in \mathbb{R}$. Moreover, $0 \in \rho(A(t))$ for each $t \in \mathbb{R}$ and the following holds

$$
\sup _{t, s \in \mathbb{R}}\|A(s) A(t)\|_{B\left(\mathbb{X}_{\alpha}, \mathbb{X}\right)}<c_{0}
$$

It holds that [23]

$$
\|y\|_{\alpha}^{t} \leq c(\alpha)\|y\|^{1-\alpha}\|A(t) y\|^{\alpha}, \quad y \in D(A(t)) .
$$

We have the following fundamental estimates for the evolution family $U(t, s)$.

Proposition 2.1 ([24]). For $x \in \mathbb{X}, 0 \leq \alpha \leq 1$ and $t>s$, the following hold.

(i) There is a constant $c(\alpha)$, such that

$$
\|U(t, s) P(s) x\|_{\alpha}^{t} \leq c(\alpha) e^{-\frac{\delta}{2}(t-s)}(t-s)^{-\alpha}\|x\| .
$$

(ii) There is a constant $m(\alpha)$, such that

$$
\left\|U_{Q}(s, t) Q(t) x\right\|_{\alpha}^{s} \leq m(\alpha) e^{-\delta(t-s)}\|x\| .
$$

Now, we recall the basic properties of weights. Let $\mathbb{U}$ be the collection of functions (weights) $\rho: \mathbb{R} \rightarrow(0, \infty)$, which are locally integrable over $\mathbb{R}$ such that $\rho>0$ almost everywhere. For $\mu \in \mathbb{U}, T>0$, and $a \in \mathbb{R}$, we set

$$
\begin{aligned}
& Q_{T}:=[-T, T], Q_{T}+a:=[-T+a, T+a], \\
& \mu\left(Q_{T}\right):=\int_{Q_{T}} \mu(x) \mathrm{d} x .
\end{aligned}
$$

We define the space of weights $\mathbb{U}_{\infty}$ by

$$
\mathbb{U}_{\infty}:=\left\{\mu \in \mathbb{U}: \inf _{x \in \mathbb{R}} \mu(x)=\mu_{0}>0 \text { and } \lim _{T \rightarrow \infty} \mu\left(Q_{T}\right)=\infty\right\}
$$

and the set of weights $\mathbb{U}_{B}$ by

$$
\mathbb{U}_{B}:=\left\{\mu \in \mathbb{U}_{\infty}: \sup _{x \in \mathbb{R}} \mu(x)=\mu_{1}<\infty\right\} .
$$

We also need the following set of weights, which makes the spaces of weighted pseudoalmost periodic functions translation-invariant,

$$
\mathbb{U}_{\infty}^{I n v}:=\left\{\mu \in \mathbb{U}_{\infty}: \lim _{x \rightarrow \infty} \frac{\mu(x+\tau)}{\mu(x)}<\infty \text { and } \lim _{T \rightarrow \infty} \frac{\mu\left(Q_{T+\tau}\right)}{\mu\left(Q_{T}\right)}<\infty \text { for all } \tau \in \mathbb{R}\right\} .
$$


Let $\mathbb{U}_{\infty}^{c}$ denote the collection of all continuous functions (weights) $\mu: \mathbb{R} \rightarrow(0, \infty)$ such that $\mu>0$ almost everywhere. Define

$$
\mathbb{U}_{\infty}^{s}:=\left\{\mu \in \mathbb{U}_{\infty}^{c} \cap \mathbb{U}_{\infty}: \lim _{x \rightarrow \infty} \frac{\mu(x+\tau)}{\mu(x)}<\infty \text { for all } \tau \in \mathbb{R}\right\} .
$$

Definition 2.2. Let $\mu, v \in \mathbb{U}_{\infty}$. One says that $\mu$ is equivalent to $v$ and denote it $\mu \prec v$, if $\frac{\mu}{v} \in \mathbb{U}_{B}$.

Let $\mu, v, \gamma \in \mathbb{U}_{\infty}$. It is clear that $\mu \prec \mu$ (reflexivity); if $\mu \prec v$, then $v \prec \mu$ (symmetry); and if $\mu \prec v$ and $v \prec \gamma$, then $\mu \prec \gamma$ (transitivity). Therefore, $\prec$ is a binary equivalence relation on $\mathbb{U}_{\infty}$.

Proposition 2.2. Let $\mu, v \in \mathbb{U}_{\infty}^{\operatorname{Inv}}$. If $\mu \prec v$, then $\sigma=\mu+v \in \mathbb{U}_{\infty}^{\mathrm{Inv}}$.

Proposition 2.3. Let $\mu, v \in \mathbb{U}_{\infty}^{s}$. Then their product $\pi=\mu \nu \in \mathbb{U}_{\infty}^{s}$. Moreover, if $\mu \prec v$, then $\sigma:=\mu+v \in \mathbb{U}_{\infty}^{s}$.

The next theorem describes all the nonconstant polynomials belonging to the set of weights $\mathbb{U}_{\infty}$.

Theorem 2.1. ([25]) If $\mu \in \mathbb{U}_{\infty}$ is a nonconstant polynomial of degree $N$, then $N$ is necessarily even $\left(N=2 n^{\prime}\right.$ for some nonnegative integer $\left.n^{\prime}\right)$. More precisely, $\mu$ can be written in the form

$$
\mu(x)=a \prod_{k=0}^{n}\left(x^{2}+a_{k} x+b_{k}\right)^{m_{k}}
$$

where $a>0$ is a constant, $a_{k}$ and $b_{k}$ are some real numbers satisfying $a_{k}^{2}-4 b_{k}<0$, and $m_{k}$ are nonnegative integers for $k=0, \ldots, n$. Furthermore, the weight $\mu$ given above belongs to $\mathbb{U}_{\infty}^{s}$.

Definition 2.3. A function $f \in C(\mathbb{R}, \mathbb{X})$ is called (Bohr) almost periodic if for each $\varepsilon>0$ there exists $l(\varepsilon)>0$ such that every interval of length $l(\varepsilon)$ contains a number $\tau$ with the property that

$$
\|f(t+\tau)-f(t)\|<\varepsilon \quad \text { for each } t \in \mathbb{R} .
$$

The collection of all almost periodic functions will be denoted $A P(\mathbb{X})$.

Definition 2.4. A function $F \in C(\mathbb{R} \times \mathbb{Y}, \mathbb{X})$ is called (Bohr) almost periodic in $t \in \mathbb{R}$ uniformly in $y \in \mathbb{Y}$ if for each $\varepsilon>0$ and any compact $K \subset \mathbb{Y}$ there exists $l(\varepsilon)$ such that every interval of length $l(\varepsilon)$ contains a number $\tau$ with the property that

$$
\|F(t+\tau, y)-F(t, y)\|<\varepsilon \quad \text { for each } t \in \mathbb{R}, y \in K \text {. }
$$

The collection of those functions is denoted by $A P(\mathbb{Y}, \mathbb{X})$. 
If $\mu, v \in \mathbb{U}_{\infty}$, we then define

$$
P A P_{0}(\mathbb{X}, \mu, v):=\left\{f \in B C(\mathbb{R}, \mathbb{X}): \lim _{T \rightarrow \infty} \frac{1}{\mu\left(Q_{T}\right)} \int_{Q_{T}}\|f(\sigma)\| v(\sigma) \mathrm{d} \sigma=0\right\} .
$$

Similarly, we define $P A P_{0}(\mathbb{Y}, \mathbb{X}, \mu, v)$ as the collection of jointly continuous functions $F$ : $\mathbb{R} \times \mathbb{Y} \rightarrow \mathbb{X}$ such that $F(\cdot, y)$ is bounded for each $y \in \mathbb{Y}$ and

$$
\lim _{T \rightarrow \infty} \frac{1}{\mu\left(Q_{T}\right)}\left\{\int_{Q_{T}}\|F(s, y)\| v(s) \mathrm{d} s\right\}=0
$$

uniformly in $y \in \mathbb{Y}$.

Definition 2.5. Let $\mu, v \in \mathbb{U}_{\infty}$. A function $f \in C(\mathbb{R}, \mathbb{X})$ is called doubly-weighted pseudo-almost periodic if it can be expressed as $f=g+\phi$, where $g \in A P(\mathbb{X})$ and $\phi \in P A P_{0}(\mathbb{X}, \mu, v)$. The collection of such functions will be denoted by $\operatorname{PAP}(\mathbb{X}, \mu, v)$.

Definition 2.6. Let $\mu, v \in \mathbb{U}_{\infty}$. A function $F \in C(\mathbb{R} \times \mathbb{Y}, \mathbb{X})$ is called doubly-weighted pseudoalmost periodic if it can be expressed as $F=G+\Phi$, where $G \in A P(\mathbb{Y}, \mathbb{X})$ and $\Phi \in P A P_{0}(\mathbb{Y}, \mathbb{X}, \mu, v)$. The collection of such functions will be denoted by $P A P(\mathbb{Y}, \mathbb{X}, \mu, v)$.

\section{Existence of a doubly-weighted mean for almost periodic functions}

Let $\mu, v \in \mathbb{U}_{\infty}$. If $f: \mathbb{R} \rightarrow \mathbb{X}$ is a bounded continuous function, we define its doubly-weighted mean, if the limit exists, by

$$
\mathcal{M}(f, \mu, v):=\lim _{T \rightarrow \infty} \frac{1}{\mu\left(Q_{T}\right)} \int_{Q_{T}} f(t) v(t) \mathrm{d} t .
$$

It is well-known that if $f \in A P(\mathbb{X})$, then its mean defined by

$$
\mathcal{M}(f):=\lim _{T \rightarrow \infty} \frac{1}{2 T} \int_{Q_{T}} f(t) \mathrm{d} t
$$

exists [26]. Consequently, for every $\lambda \in \mathbb{R}$, the following limit

$$
a(f, \lambda):=\lim _{T \rightarrow \infty} \frac{1}{2 T} \int_{Q_{T}} f(t) e^{-i \lambda t} \mathrm{~d} t
$$

exists and is called the Bohr transform of $f$.

It is also well-known that $a(f, \lambda)$ is nonzero at most at countably many points [26]. The set defined by

$$
\sigma_{b}(f):=\{\lambda \in \mathbb{R}: a(f, \lambda) \neq 0\}
$$


is called the Bohr spectrum of $f$ [27].

In [12], the original question which consists of the existence of a weighted mean for almost periodic functions was raised. In particular, the authors have shown through an example that there exist weights for which a weighted mean for almost periodic functions did not exist. In this section we investigate the broader question, which consists of the existence of a doubly-weighted mean for almost periodic functions. Namely, we give some sufficient conditions, which guarantee the existence of a doubly-weighted mean for almost periodic functions. Moreover, under those conditions, it will be shown that the doubly-weighted mean and the classical (Bohr) mean are proportional. Further, it will be shown that if the doubly-weighted Bohr spectrum of an almost periodic function exists, then it is either empty or coincides with the Bohr spectrum of that function. In [1], it is proved that for $\mu, v \in \mathbb{U}_{\infty}$ and $f: \mathbb{R} \rightarrow \mathbb{X}$ an almost periodic function such that

$$
\lim _{T \rightarrow \infty} \frac{v\left(Q_{T}\right)}{\mu\left(Q_{T}\right)}=\theta_{\mu \nu}, \quad \lim _{T \rightarrow \infty}\left|\frac{1}{\mu\left(Q_{T}\right)} \int_{Q_{T}} e^{i \lambda t} v(t) \mathrm{d} t\right|=0,
$$

for all $0 \neq \lambda \in \sigma_{b}(f)$, the doubly-weighted mean of $f$,

$$
\mathcal{M}(f, \mu, v)=\lim _{T \rightarrow \infty} \frac{1}{\mu\left(Q_{T}\right)} \int_{Q_{T}} f(t) v(t) \mathrm{d} t
$$

exists. Furthermore, $\mathcal{M}(f, \mu, v)=\theta_{\mu v} \mathcal{M}(f)$.

Consider the set of weights $\mathbb{U}_{\infty}^{0}$ defined by

$$
\mathbb{U}_{\infty}^{0}=\left\{\mu \in \mathbb{U}_{\infty}: D_{\tau}:=\lim _{|t| \rightarrow \infty} \frac{\mu\left(Q_{t+\tau}\right)}{\mu\left(Q_{t}\right)}<\infty \text { for all } \tau \in \mathbb{R}\right\}
$$

Setting $C_{\tau}=\lim _{|t| \rightarrow \infty} \frac{\mu\left(Q_{t}+\tau\right)}{\mu\left(Q_{t}\right)}$, one can easily see that $C_{\tau} \leq D_{\tau}<\infty$ for all $\tau \in \mathbb{R}$.

Definition 3.1. ([1]) Fix $\mu, v \in \mathbb{U}_{\infty}$ and suppose that $\lim _{T \rightarrow \infty} \frac{v\left(Q_{T}\right)}{\mu\left(Q_{T}\right)}=\theta_{\mu v}$. If $f: \mathbb{R} \rightarrow \mathbb{X}$ is an almost periodic function such that (3.1) holds, we then define its doubly-weighted Bohr transform as

$$
\widehat{a}_{\mu \nu}(f)(\lambda):=\lim _{T \rightarrow \infty} \frac{1}{\mu\left(Q_{T}\right)} \int_{Q_{T}} f(t) e^{-i \lambda t} \nu(t) \mathrm{d} t \quad \text { for all } \lambda \in \mathbb{R} .
$$

Now since $t \rightarrow g_{\lambda}(t):=f(t) e^{-i \lambda t} \in A P(\mathbb{X})$ it follows that

$$
\widehat{a}_{\mu \nu}(f)(\lambda)=\theta_{\mu \nu} \mathcal{M}\left(f(\cdot) e^{-i \lambda \cdot}\right)=\theta_{\mu \nu} a(f, \lambda) .
$$

That is, under (3.1),

$$
\begin{aligned}
\widehat{a}_{\mu v}(f)(\lambda) & :=\lim _{T \rightarrow \infty} \frac{1}{\mu\left(Q_{T}\right)} \int_{Q_{T}} f(t) e^{-i \lambda t} v(t) \mathrm{d} t \\
& =\theta_{\mu \nu} \lim _{T \rightarrow \infty} \frac{1}{2 T} \int_{Q_{T}} f(t) e^{-i \omega t} \mathrm{~d} t=\theta_{\mu \nu} a(f, \lambda), \quad \text { for all } \lambda \in \mathbb{R} .
\end{aligned}
$$




\section{Main results}

In this section, we fix the two weights $\mu, v \in \mathbb{U}_{\infty}$ such that $P A P(\mathbb{X}, \mu, v)$ is translationinvariant and that

$$
\inf _{T>0}\left[\frac{v\left(Q_{T}\right)}{\mu\left(Q_{T}\right)}\right]=\delta_{0}>0 .
$$

Assume that $R(\omega, A(\cdot)) \in A P(B(\mathbb{X}))$ and that there there exists a function $H:[0, \infty) \mapsto[0, \infty)$ with $H \in L^{1}[0, \infty)$ such that for every $\varepsilon>0$ there exists $l(\varepsilon)$ such that every interval of length $l(\varepsilon)$ contains a $\tau$ with the property

$$
\|A(t+\tau) U(t+\tau, s+\tau) P(s+\tau)-A(t) U(t, s) P(s)\|_{B\left(\mathbb{X}, \mathbb{X}_{\alpha}\right)} \leq \varepsilon H(t-s)
$$

for all $t, s \in \mathbb{R}$ with $t>s$.

Assume further that there exists $0 \leq \alpha<\beta<1$ such that

$$
\mathbb{X}_{\alpha}^{t}=\mathbb{X}_{\alpha}, \quad \mathbb{X}_{\beta}^{t}=\mathbb{X}_{\beta} \text { for all } t \in \mathbb{R} \text {, with uniform equivalent norm, }
$$

where

$$
\begin{aligned}
& \mathbb{X}_{\alpha}^{A}=\left\{x \in \mathbb{X}, \sup _{r>0}\left\|r^{\alpha}(A-\omega) R(r, A-\omega) x\right\|<\infty\right\}, \\
& \mathbb{X}_{\beta}^{A}=\left\{x \in \mathbb{X}, \sup _{r>0}\left\|r^{\beta}(A-\omega) R(r, A-\omega) x\right\|<\infty\right\} .
\end{aligned}
$$

Furthermore, if $0 \leq \alpha<\beta<1$, then we let $k=k(\alpha, \beta), k_{1}=k_{1}(\alpha), k_{2}=k_{2}(\beta)$ be the bounds of the continous injections

$$
X_{\beta} \hookrightarrow X_{\alpha}, X_{\alpha} \hookrightarrow X, X_{\beta} \hookrightarrow X
$$

that is

$$
\begin{aligned}
& \|u\|_{\alpha} \leq k\|u\|_{\beta} \text { for each } u \in \mathbb{X}_{\beta},\|u\| \leq k_{1}\|u\|_{\alpha} \text { for each } u \in \mathbb{X}_{\alpha}, \\
& \text { and }\|u\| \leq k_{2}\|u\|_{\beta} \text { for each } u \in \mathbb{X}_{\beta} .
\end{aligned}
$$

For $\mu, v \in \mathbb{U} U_{\infty}$ and $0<\alpha<\beta<1$ let $f: \mathbb{R} \times \mathbb{X} \mapsto \mathbb{X}_{\beta}$ be in $P A P\left(\mathbb{X}, \mathbb{X}_{\beta}, \mu, v\right)$ such that

$$
\|f(t, u)-f(t, v)\|_{\beta} \leq K_{f}\|u-v\|
$$

for all $u, v \in \mathbb{X}$ and $t \in \mathbb{R}$. with $K_{f}>0$.

To state our main result in this paper, we need the following extra-hypotheses

(H1) Let $\mu, v \in \mathbb{U} U_{\infty}$. We suppose $g: \mathbb{R} \times \mathbb{X} \mapsto \mathbb{X}$ belongs to $P A P(\mathbb{X}, \mathbb{X}, \mu, v)$ and there exists $K_{g}>0$ such that

$$
\|g(t, u)-g(t, v)\| \leq K_{g}\|u-v\|,
$$

for all $u, v \in \mathbb{X}$ and $t \in \mathbb{R}$. 
(H2) We suppose that the linear operators $B(t), C(t): \mathbb{X}_{\alpha} \mapsto \mathbb{X}$ for all $t \in \mathbb{R}$, are bounded and set

$$
\omega:=\max \left(\sup _{t \in \mathbb{R}}\|B(t)\|_{B\left(\mathbb{X}_{\alpha}, \mathbb{X}\right)}, \sup _{t \in \mathbb{R}}\|C(t)\|_{B\left(\mathbb{X}_{\alpha}, \mathbb{X}\right)}\right) .
$$

Furthermore, $t \mapsto B(t)$ and $t \mapsto C(t)$ are almost periodic.

To study the existence and uniqueness of doubly-weighted pseudo-almost periodic solutions to (1.1) we first recall the notion of mild solution.

Definition 4.1. A function $u: \mathbb{R} \mapsto \mathbb{X}_{\alpha}$ is said to be a mild solution to equation (1.2) provided that the function $\tau \mapsto A(\tau) U(t, \tau) P(\tau) f(\tau, B(\tau) u(\tau))$ is integrable on $(s, t)$,

$$
\tau \mapsto A(\tau) U(t, \tau) Q(\tau) f(\tau, B(\tau) u(\tau))
$$

is integrable on $(t, s)$ and

$$
\begin{aligned}
u(t)=-f( & , B(t) u(t))+U(t, s)(u(s)+f(s, B(s) u(s))) \\
& -\int_{s}^{t} A(\tau) U(t, \tau) P(\tau) f(\tau, B(\tau) u(\tau)) \mathrm{d} \tau \\
& +\int_{t}^{s} A(\tau) U_{Q}(t, \tau) Q(\tau) f(\tau, B(\tau) u(\tau)) \mathrm{d} \tau \\
& +\int_{s}^{t} U(t, \tau) P(\tau) g(\tau, C(\tau) u(\tau)) \mathrm{d} \tau \\
& -\int_{t}^{s} U_{Q}(t, \tau) Q(\tau) g(\tau, C(\tau) u(\tau)) \mathrm{d} \tau
\end{aligned}
$$

for $t \geq s$ and for all $t, s \in \mathbb{R}$.

In [28], it is shown under more hypotheses than (H1) and (H2) that a mild solution $u$ of (1.2) on $\mathbb{R}$ satisfies for all $t \in \mathbb{R}$,

$$
\begin{aligned}
u(t)=-f( & t, B(t) u(t))-\int_{-\infty}^{t} A(s) U(t, s) P(s) f(s, B(s) u(s)) \mathrm{d} s \\
& +\int_{t}^{\infty} A(s) U_{Q}(t, s) Q(s) f(s, B(s) u(s)) \mathrm{d} s \\
& +\int_{-\infty}^{t} U(t, s) P(s) g(s, C(s) u(s)) \mathrm{d} s \\
& -\int_{t}^{\infty} U_{Q}(t, s) Q(s) g(s, C(s) u(s)) \mathrm{d} s .
\end{aligned}
$$

Throughout the rest of the paper we denote by $\Gamma_{1}, \Gamma_{2}, \Gamma_{3}$ and $\Gamma_{4}$, the nonlinear integral operators defined by

$$
\left(\Gamma_{1} u\right)(t):=\int_{-\infty}^{t} A(s) U(t, s) P(s) f(s, B(s) u(s)) \mathrm{d} s,
$$




$$
\begin{aligned}
& \left(\Gamma_{2} u\right)(t):=\int_{t}^{\infty} A(s) U_{Q}(t, s) Q(s) f(s, B(s) u(s)) \mathrm{d} s, \\
& \left(\Gamma_{3} u\right)(t):=\int_{-\infty}^{t} U(t, s) P(s) g(s, C(s) u(s)) \mathrm{d} s, \\
& \left(\Gamma_{4} u\right)(t):=\int_{t}^{\infty} U_{Q}(t, s) Q(s) g(s, C(s) u(s)) \mathrm{d} s .
\end{aligned}
$$

We now state our main result in this paper.

Theorem 4.1. If assumptions (H1)-(H2) hold and $\kappa<1$ the equation (1.2) has a unique doublyweighted pseudo-almost periodic mild solution, where

$$
\begin{array}{rl}
\kappa=k_{1} K_{G} & \mathcal{O}\left[\delta^{-1} m(\alpha)+c(\alpha) 2^{1-\alpha} \delta^{\alpha-1} \Gamma(1-\alpha)\right] \\
+ & k_{1} K_{F} \mathcal{\omega}\left[k+\delta^{-1} m(\alpha, \beta)+4^{1-\alpha} \delta^{\alpha-1} n(\alpha, \theta) \Gamma(1-\alpha)\right] .
\end{array}
$$

The proof of this results reposes on the following technique and preliminary lemmas.

Lemma 4.1. Under the hypotheses (H6) and (H7), the following assertion holds.

- if $u \in P A P\left(\mathbb{R}, \mathbb{X}_{\alpha}, \mu, v\right)$, then $C() u.(.) \in P A P(\mathbb{R}, \mathbb{X}, \mu, v)$.

Proof. Let $u \in P A P\left(\mathbb{R}, \mathbb{X}_{\alpha}, \mu, v\right)$. Then $u=u_{1}+u_{2}$ where $u_{1} \in A P\left(\mathbb{R}, \mathbb{X}_{\alpha}\right), u_{2} \in P A P_{0}\left(\mathbb{R}, \mathbb{X}_{\alpha}, \mu, v\right)$. We have

$$
C(t) u(t)=C(t) u_{1}(t)+C(t) u_{2}(t), \quad \text { for all } t \in \mathbb{R} .
$$

Since $u_{1} \in A P\left(\mathbb{R}, \mathbb{X}_{\alpha}\right)$, for every $\epsilon>0$ there exists $l_{\epsilon}$ such that every interval of length $l_{\epsilon}$ contains a $\tau$ such

$$
\left\|u_{1}(t+\tau)-u_{1}(t)\right\|_{\alpha}<\frac{\epsilon}{\left(\sup _{t \in \mathbb{R}}\left\|u_{1}(t)\right\|_{\alpha}+\omega\right)}, \quad t \in \mathbb{R} .
$$

Similarly, since $C(t) \in A P\left(B\left(\mathbb{X}_{\alpha}, \mathbb{X}\right)\right)$, we have

$$
\|C(t+\tau)-C(t)\|_{B\left(\mathbb{X}_{\alpha}, \mathbb{X}\right)}<\frac{\epsilon}{\left(\sup _{t \in \mathbb{R}}\left\|u_{1}(t)\right\|_{\alpha}+\boldsymbol{\omega}\right)}, \quad t \in \mathbb{R} .
$$

Now

$$
\begin{aligned}
& \left\|C(t+\tau) u_{1}(t+\tau)-C(t) u_{1}(t)\right\| \\
\leq & \left\|[C(t+\tau)-C(t)] u_{1}(t+\tau)\right\|+\|\| C(t)\left[u_{1}(t+\tau)-u_{1}(t)\right] \| \\
\leq & \|C(t+\tau)-C(t)\|_{B\left(\mathbb{X}_{\alpha}, \mathbb{X}\right)}\left\|u_{1}(t+\tau)\right\|_{\alpha}+\omega\left\|u_{1}(t+\tau)-u_{1}(t)\right\|_{\alpha} \leq \epsilon,
\end{aligned}
$$

and hence $t \mapsto C(t) u_{1}(t)$ belongs to $A P(\mathbb{R}, \mathbb{X})$. 
To complete the proof, it suffices prove that $t \mapsto C(t) u_{2}(t)$ belongs to $P A P_{0}(\mathbb{R}, \mathbb{X}, \mu, v)$, we have

$$
\frac{1}{\mu\left(Q_{T}\right)} \int_{-r}^{r}\left\|C(t) u_{2}(t)\right\| \mathrm{d} v(t) \leq \frac{\mathscr{\omega}}{\left.\mu\left(Q_{T}\right)\right)} \int_{-T}^{T}\left\|u_{2}(t)\right\|_{\alpha} \mathrm{d} v(t)
$$

and hence

$$
\lim _{r \rightarrow \infty} \frac{1}{\mu\left(Q_{T}\right)} \int_{-T}^{T}\left\|C(t) u_{2}(t)\right\| \mathrm{d} v(t)=0
$$

In [24], it is proved that for $0 \leq \theta<\alpha<\beta<1$ with $2 \alpha>\theta+1$, then there exist two constants $m(\alpha, \beta), n(\alpha, \theta)>0$ such that

$$
\begin{array}{ll}
\left\|A(s) U_{Q}(t, s) Q(s) x\right\|_{\alpha} \leq m(\alpha, \beta) e^{\delta(t-s)}\|x\|_{\beta}, & \text { for } t \leq s, \\
\|A(s) U(t, s) P(s) x\|_{\alpha} \leq n(\alpha, \theta)(t-s)^{-\alpha} e^{-\frac{\delta}{4}(t-s)}\|x\|_{\beta}, & \text { for } t>s .
\end{array}
$$

Lemma 4.2. Assume that $(\mathrm{H} 2)$ hold, then the integral operators $\Gamma_{1}$ and $\Gamma_{2}$ defined above map $\operatorname{PAP}\left(\mathbb{X}_{\alpha}, \mu, v\right)$ into itself.

Proof. Let $u \in P A P\left(\mathbb{X}_{\alpha}, \mu, v\right)$. From Lemma 4.1 it follows that the function $t \mapsto B(t) u(t)$ belongs to $\operatorname{PAP}(\mathbb{X}, \mu, v)$. It follows that $\psi(\cdot)=f(\cdot, B u(\cdot))$ is in $\operatorname{PAP}\left(\mathbb{X}_{\beta}, \mu, v\right)$ whenever $u \in P A P\left(\mathbb{X}_{\alpha}, \mu, v\right)$. In particular,

$$
\|\psi\|_{\infty, \beta}=\sup _{t \in \mathbb{R}}\|f(t, B u(t))\|_{\beta}<\infty .
$$

Since $\psi(\cdot)=f(\cdot, B u(\cdot))$ is in $P A P\left(\mathbb{X}_{\beta}, \mu, v\right)$ then $\psi=\phi_{1}+\phi_{2}$, where $\phi_{1} \in A P\left(\mathbb{R}, \mathbb{X}_{\beta}\right)$ and $\phi_{2} \in P A P_{0}\left(\mathbb{R}, \mathbb{X}_{\beta}, \mu, v\right)$, that is, $\Gamma_{1} \psi=\Xi\left(\phi_{1}\right)+\Xi\left(\phi_{2}\right)$ where

$$
\begin{aligned}
& \Xi \phi_{1}(t):=\int_{-\infty}^{t} A(s) U(t, s) P(s) \phi_{1}(s) \mathrm{d} s, \\
& \Xi \phi_{2}(t):=\int_{-\infty}^{t} A(s) U(t, s) P(s) \phi_{2}(s) \mathrm{d} s .
\end{aligned}
$$

Firstly, we show that $\Xi \phi_{1} \in B C\left(\mathbb{R}, \mathbb{X}_{\beta}\right)$, using estimate (4.4), we obtain

$$
\begin{aligned}
\left\|\Xi \phi_{1}(t)\right\|_{\beta} & \leq \int_{-\infty}^{t}\left\|A(s) U(t, s) P(s) \phi_{1}(s)\right\| \mathrm{d} s \\
& \leq n(\alpha, \theta) \int_{-\infty}^{t}(t-s)^{-\alpha} e^{-\frac{\delta}{4}(t-s)}\left\|\phi_{1}\right\|_{\beta} \mathrm{d} s \\
& \leq n(\alpha, \theta)\left(\frac{\delta}{4}\right)^{1-\alpha} \Gamma(1-\alpha)\left\|\phi_{1}\right\|_{\beta} .
\end{aligned}
$$

Then $\Xi \phi_{1} \in B C\left(\mathbb{R}, \mathbb{X}_{\beta}\right)$. 
Next, we prove that $\Xi\left(\phi_{1}\right) \in A P\left(\mathbb{R}, \mathbb{X}_{\alpha}\right)$. Since $\phi_{1} \in A P\left(\mathbb{R}, \mathbb{X}_{\beta}\right)$, then for every $\epsilon>0$ there exists $l(\epsilon)>0$ such that every interval of length $l(\epsilon)$ contains a $\tau$ with the property

$$
\left\|\phi_{1}(t+\tau)-\phi_{1}(t)\right\|_{\beta}<\epsilon v_{1} \text { for each } t \in \mathbb{R},
$$

where $v_{1}=\frac{4^{\alpha-1} \delta^{1-\alpha}}{n(\alpha, \mu) \Gamma(1-\alpha)}$. Now, we have

$$
\begin{aligned}
& \Xi \phi_{1}(t+\tau)-\Xi \phi_{1}(t)=\int_{-\infty}^{t+\tau} A(s) U(t+\tau, s) P(s) \phi_{1}(s) \mathrm{d} s-\int_{-\infty}^{t} A(s) U(t, s) P(s) \phi_{1}(s) \mathrm{d} s \\
= & \int_{-\infty}^{t} A(s+\tau) U(t+\tau, s+\tau)+P(s+\tau)\left(\phi_{1}(s+\tau)-\phi_{1}(s)\right) \mathrm{d} s \\
& \quad+\int_{-\infty}^{t}(A(s+\tau) U(t+\tau, s+\tau) P(s+\tau)-A(s) U(t, s) P(s)) \phi_{1}(s) \mathrm{d} s .
\end{aligned}
$$

Using equation (4.4) it follows that

$$
\left\|\int_{-\infty}^{t} A(s+\tau) U(t+\tau, s+\tau) P(s+\tau)\left(\phi_{1}(s+\tau)-\phi_{1}(s)\right) \mathrm{d} s\right\|_{\alpha} \leq \epsilon .
$$

Similarly, using assumption (H2), it follows that

$$
\left\|\int_{-\infty}^{t}[A(s+\tau) U(t+\tau, s+\tau) P(s+\tau)-A(s) U(t, s) P(s)] \phi_{1}(s) \mathrm{d} s\right\|_{\alpha} \leq \epsilon\|H\|_{L^{1}}\left\|\phi_{1}\right\|_{\infty},
$$

where $\|H\|_{L^{1}}=\int_{0}^{\infty} H(s) \mathrm{d} s<\infty$. Finally, we have

$$
\left\|\Xi\left(\phi_{1}\right)(t+\tau)-\Xi\left(\phi_{1}\right)(t)\right\|_{\alpha} \leq\left(1+\|H\|_{L^{1}}\left\|\phi_{1}\right\|_{\infty}\right) \epsilon, \quad \text { for each } t \in \mathbb{R},
$$

and hence $\Xi\left(\phi_{1}\right) \in A P\left(\mathbb{R}, \mathbb{X}_{\alpha}\right)$.

Now, we show that $\Xi\left(\phi_{2}\right) \in B C\left(\mathbb{R}, \mathbb{X}_{\beta}\right)$. Using estimate (4.4) and adopt the technique developed for the function $\Xi\left(\phi_{1}\right)$.

To complete the proof, we will prove that $\Xi\left(\phi_{2}\right) \in P A P_{0}\left(\mathbb{R}, \mathbb{X}_{\beta}, \mu\right)$. So, let $T>0$. Again from Eq. (4.4), we have

$$
\begin{aligned}
& \frac{1}{\mu\left(Q_{T}\right)} \int_{-T}^{T}\left\|\left(\Xi \phi_{2}\right)(t)\right\|_{\alpha} \mathrm{d} v(t) \\
\leq & \frac{1}{\mu\left(\left[Q_{T}\right)\right.} \int_{-T}^{T} \int_{0}^{+\infty}\left\|A(t-s) U(t, t-s) P(t-s) \phi_{2}(t-s)\right\|_{\alpha} \mathrm{d} s \mathrm{~d} v(t) \\
\leq & \frac{n(\alpha, \theta)}{\mu\left(Q_{T}\right)} \int_{-T}^{T} \int_{0}^{+\infty} s^{-\alpha} e^{-\frac{\delta}{4} s}\left\|\phi_{2}(t-s)\right\|_{\beta} \mathrm{d} s \mathrm{~d} v(t) \\
\leq & n(\alpha, \theta) \int_{0}^{+\infty} s^{-\alpha} e^{-\frac{\delta}{4} s}\left(\frac{1}{\mu\left(Q_{T}\right)} \int_{-T}^{T}\left\|\phi_{2}(t-s)\right\|_{\beta} \mathrm{d} v(t)\right) \mathrm{d} s .
\end{aligned}
$$


Now

$$
\lim _{T \rightarrow \infty} \frac{1}{\mu\left(Q_{T}\right)} \int_{-T}^{T}\left\|\phi_{2}(t-s)\right\|_{\beta} \mathrm{d} v(t)=0 .
$$

The function $t \mapsto \phi_{2}(t-s) \in P A P_{0}\left(\mathbb{R}, \mathbb{X}_{\beta}, \mu, v\right)$ for every $s \in \mathbb{R}$. To completes the proof, using the Lebesgue's dominated convergence theorem.

The proof for $\Gamma_{2} u(\cdot)$ is similar to that of $\Gamma_{1} u(\cdot)$ except that one makes use of equation (4.3) instead of equation (4.4).

Lemma 4.3. If assumptions (H1)-(H2), hold, then the integral operators $\Gamma_{3}$ and $\Gamma_{4}$ defined above map $P A P\left(\mathbb{R}, \mathbb{X}_{\alpha}, \mu, v\right)$ into itself.

Proof. Let $u \in P A P\left(\mathbb{R}, \mathbb{X}_{\alpha}, \mu, v\right)$. From Lemma 4.1 it follows that

$$
C(\cdot) u(\cdot) \in P A P(\mathbb{R}, \mathbb{X}, \mu, v) .
$$

Setting $h(t)=g(t, C u(t))$. It is straightforward that $h \in P A P(\mathbb{R}, \mathbb{X}, \mu, v)$. So, write next $h=\psi_{1}+\psi_{2}$, where $\psi_{1} \in A P(\mathbb{R}, \mathbb{X})$ and $\psi_{2} \in P A P_{0}(\mathbb{R}, \mathbb{X}, \mu, v)$, that is, $\Gamma_{3} h=\Xi\left(\psi_{1}\right)+\Xi\left(\psi_{2}\right)$ where

$$
\begin{aligned}
& \Xi \psi_{1}(t):=\int_{-\infty}^{t} U(t, s) P(s) \psi_{1}(s) \mathrm{d} s, \\
& \Xi \psi_{2}(t):=\int_{-\infty}^{t} U(t, s) P(s) \psi_{2}(s) \mathrm{d} s .
\end{aligned}
$$

Firstly, we show that $\Xi \psi_{1} \in B C\left(\mathbb{R}, \mathbb{X}_{\beta}\right)$, using estimate (2.7), we obtain

$$
\begin{aligned}
\left\|\Xi \psi_{1}(t)\right\|_{\beta} & \leq \int_{-\infty}^{t}\left\|U(t, s) P(s) \psi_{1}(s)\right\| \mathrm{d} s \\
& \leq c(\alpha) \int_{-\infty}^{t}(t-s)^{-\alpha} e^{-\frac{\delta}{2}(t-s)}\left\|\psi_{1}\right\|_{\beta} \mathrm{d} s \\
& \leq c(\alpha)\left(\frac{\delta}{2}\right)^{1-\alpha} \Gamma(1-\alpha)\left\|\psi_{1}\right\|_{\beta} .
\end{aligned}
$$

Then $\Xi \psi_{1} \in B C\left(\mathbb{R}, \mathbb{X}_{\beta}\right)$.

Next, we prove that $\Xi\left(\psi_{1}\right) \in A P\left(\mathbb{R}, \mathbb{X}_{\alpha}\right)$. Since $\psi_{1} \in A P\left(\mathbb{R}, \mathbb{X}_{\beta}\right)$, then for every $\epsilon>0$ there exists $l(\epsilon)>0$ such that every interval of length $l(\epsilon)$ contains a $\tau$ with the property

$$
\left\|\psi_{1}(t+\tau)-\psi_{1}(t)\right\|_{\beta}<\epsilon \eta, \quad \text { for each } t \in \mathbb{R},
$$

where $\eta=\frac{1}{2^{\alpha} \delta^{1-\alpha} c(\alpha) \Gamma(1-\alpha)}$. Now

$$
\Xi \psi_{1}(t+\tau)-\Xi \psi_{1}(t)=\int_{-\infty}^{t+\tau} U(t+\tau, s) P(s) \psi_{1}(s) \mathrm{d} s-\int_{-\infty}^{t} U(t, s) P(s) \psi_{1}(s) \mathrm{d} s
$$




$$
\begin{array}{rl}
=\int_{-\infty}^{t} & U(t+\tau, s+\tau) P(s+\tau)\left(\psi_{1}(s+\tau)-\psi_{1}(s)\right) \mathrm{d} s \\
& \quad+\int_{-\infty}^{t}(U(t+\tau, s+\tau) P(s+\tau)-U(t, s) P(s)) \psi_{1}(s) \mathrm{d} s .
\end{array}
$$

Using equation (2.7) it follows that

$$
\left\|\int_{-\infty}^{t} U(t+\tau, s+\tau) P(s+\tau)\left(\phi_{1}(s+\tau)-\phi_{1}(s)\right) \mathrm{d} s\right\|_{\alpha} \leq \frac{\epsilon}{2} .
$$

Similarly, using assumption (H2). Let $\epsilon>0$, from [36] we know that

$$
r \rightarrow \Gamma(t+r, s+r) \in A P(\mathbb{B}(\mathbb{X})), \quad \text { for } t, s \in \mathbb{R},
$$

where we may take the same almost periods for $t, s$ with $\|t-s\| \leq h>0$. Hence, there exists $l(\epsilon)>0$ such that every interval of length $l(\epsilon)$ contains a number $\tau>0$ with the property that, for $t \in \mathbb{R}, \sigma>0$ :

$$
\|U(t+\tau, s+\tau) P(s+\tau)-U(t, s) P(s)\| \leq \frac{\epsilon}{2\left\|\psi_{1}\right\|_{\beta}} .
$$

Therefore,

$$
\left\|\Xi\left(\psi_{1}\right)(t+\tau)-\Xi\left(\psi_{1}\right)(t)\right\|_{\alpha} \leq \epsilon
$$

for each $t \in \mathbb{R}$, and hence $\Xi\left(\psi_{1}\right) \in A P\left(\mathbb{R}, \mathbb{X}_{\alpha}\right)$.

Now, we show that $\Xi\left(\psi_{2}\right) \in B C\left(\mathbb{R}, \mathbb{X}_{\beta}\right)$. Using estimate (2.8) and adopt the technique developed for the function $\Xi\left(\psi_{1}\right)$.

Finally, to complete the proof, we will prove that $\Xi\left(\psi_{2}\right) \in P A P_{0}\left(\mathbb{R}, \mathbb{X}_{\beta}, \mu, v\right)$. Now, let $T>0$. Again from equation (2.8), we have

$$
\begin{aligned}
& \frac{1}{\mu\left(Q_{T}\right)} \int_{-T}^{T}\left\|\left(\Xi \psi_{2}\right)(t)\right\|_{\alpha} \mathrm{d} v(t) \\
\leq & \frac{1}{\mu\left(Q_{T}\right)} \int_{-T}^{T} \int_{0}^{+\infty}\left\|U(t, t-s) P(t-s) \psi_{2}(t-s)\right\|_{\alpha} \mathrm{d} s \mathrm{~d} v(t) \\
\leq & \frac{c(\alpha)}{\mu\left(Q_{T}\right)} \int_{-T}^{T} \int_{0}^{+\infty} s^{-\alpha} e^{-\frac{\delta}{2} s}\left\|\psi_{2}(t-s)\right\|_{\beta} \mathrm{d} s \mathrm{~d} v(t) \\
\leq & c(\alpha) \int_{0}^{+\infty} s^{-\alpha} e^{-\frac{\delta}{2} s}\left(\frac{1}{\mu\left(Q_{T}\right)} \int_{-T}^{T}\left\|\psi_{2}(t-s)\right\|_{\beta} \mathrm{d} v(t)\right) \mathrm{d} s .
\end{aligned}
$$

Now

$$
\lim _{T \rightarrow \infty} \frac{1}{\mu\left(Q_{T}\right)} \int_{-T}^{T}\left\|\psi_{2}(t-s)\right\|_{\beta} \mathrm{d} v(t)=0 .
$$

The function $t \mapsto \psi_{2}(t-s) \in P A P_{0}\left(\mathbb{R}, \mathbb{X}_{\beta}, \mu, v\right)$ for every $s \in \mathbb{R}$. To completes the proof, using the Lebesgue's dominated convergence theorem.

The proof for $\Gamma_{4} u(\cdot)$ is similar to that of $\Gamma_{3} u(\cdot)$ except that one makes use of Eq. (2.7) instead of Eq. (2.8). 
Proof of Theorem 4.1: Consider the nonlinear operator $\mathbb{M}$ defined on $P A P\left(\mathbb{X}_{\alpha}, \mu\right)$ by

$$
\begin{aligned}
\mathbb{M} u(t)=-F( & t, B(t) u(t))-\int_{-\infty}^{t} A(s) U(t, s) P(s) F(s, B(s) u(s)) \mathrm{d} s \\
& +\int_{t}^{\infty} A(s) U_{Q}(t, s) Q(s) F(s, B(s) u(s)) \mathrm{d} s+\int_{-\infty}^{t} U(t, s) P(s) G(s, C(s) u(s)) \mathrm{d} s \\
& -\int_{t}^{\infty} U_{Q}(t, s) Q(s) G(s, C(s) u(s)) \mathrm{d} s,
\end{aligned}
$$

for each $t \in \mathbb{R}$. Next, in view of Lemma (4.2) and Lemma (4.3), it follows that $\mathbb{M}$ maps $P A P\left(\mathbb{R}, \mathbb{X}_{\alpha}, \mu\right)$ into itself. To complete the proof one has to show that $\mathbb{M}$ is a contraction map on $P A P\left(\mathbb{R}, \mathbb{X}_{\alpha}, \mu\right)$. Let $u, v \in P A P\left(\mathbb{R}, \mathbb{X}_{\alpha}, \mu\right)$. Firstly, we have

$$
\begin{aligned}
& \left\|\Gamma_{1}(v)(t)-\Gamma_{1}(u)(t)\right\|_{\alpha} \\
\leq & n(\alpha, \theta) \int_{-\infty}^{t}(t-s)^{-\alpha} e^{-\frac{\delta}{4}(t-s)}\|F(s, B(s) v(s))-F(s, B(s) u(s))\|_{\beta} \mathrm{d} s \\
\leq & n(\alpha, \theta) K_{F} \omega \int_{-\infty}^{t}(t-s)^{-\alpha} e^{-\frac{\delta}{4}(t-s)}\|v(s)-u(s)\| \mathrm{d} s \\
\leq & n(\alpha, \theta) K_{F} \omega \int_{-\infty}^{t} k_{1}(t-s)^{-\alpha} e^{-\frac{\delta}{4}(t-s)}\|v(s)-u(s)\|_{\alpha} \mathrm{d} s \\
\leq & n(\alpha, \theta) K_{F} k_{1} \omega\|v-u\|_{\infty, \alpha} \int_{-\infty}^{t}(t-s)^{-\alpha} e^{-\frac{\delta}{4}(t-s)} \mathrm{d} s \\
= & 4^{1-\alpha} \delta^{\alpha-1} n(\alpha, \theta) \Gamma(1-\alpha) K_{F} \omega k_{1}\|v-u\|_{\infty, \alpha} .
\end{aligned}
$$

Next, we have

$$
\begin{aligned}
& \left\|\Gamma_{2}(v)(t)-\Gamma_{2}(u)(t)\right\|_{\alpha} \\
\leq & m(\alpha, \beta) \int_{t}^{\infty} e^{\delta(t-s)}\|F(s, B(s) v(s))-F(s, B(s) u(s))\|_{\beta} \mathrm{d} s \\
\leq & m(\alpha, \beta) K_{F} \int_{t}^{+\infty} e^{\delta(t-s)}\|B(s) v(s)-B(s) u(s)\| \mathrm{d} s \\
\leq & m(\alpha, \beta) K_{F} \omega \int_{t}^{+\infty} k_{1} e^{\delta(t-s)}\|v(s)-u(s)\|_{\alpha} \mathrm{d} s \\
\leq & m(\alpha, \beta) K_{F} \omega k_{1}\|v-u\|_{\infty, \alpha} \int_{t}^{+\infty} e^{\delta(t-s)} \mathrm{d} s \\
= & \delta^{-1} m(\alpha, \beta) K_{F} \omega k_{1}\|v-u\|_{\infty, \alpha} .
\end{aligned}
$$

Now, we have

$$
\begin{aligned}
& \left\|\Gamma_{3}(v)(t)-\Gamma_{3}(u)(t)\right\|_{\alpha} \\
\leq & \int_{-\infty}^{t}\|U(t, s) P(s)[G(s, C(s) v(s))-G(s, C(s) u(s))]\|_{\alpha} \mathrm{d} s
\end{aligned}
$$




$$
\begin{aligned}
& \leq c(\alpha) \int_{-\infty}^{t}(t-s)^{-\alpha} e^{-\frac{\delta}{2}(t-s)}\|C(s) v(s)-C(s) u(s)\| \mathrm{d} s \\
& \leq \omega k_{1} K_{G} c(\alpha) \int_{-\infty}^{t}(t-s)^{-\alpha} e^{-\frac{\delta}{2}(t-s)}\|v(s)-u(s)\|_{\alpha} \mathrm{d} s \\
& \leq K_{G} \omega k_{1} c(\alpha) 2^{1-\alpha} \delta^{\alpha-1} \Gamma(1-\alpha)\|v-u\|_{\infty, \alpha} .
\end{aligned}
$$

Finally, we have

$$
\begin{aligned}
& \left\|\Gamma_{4}(v)(t)-\Gamma_{4}(u)(t)\right\|_{\alpha} \\
\leq & m(\alpha) \int_{t}^{\infty} e^{\delta(t-s)}\|G(s, C(s) v(s))-G(s, C(s) u(s))\| \mathrm{d} s \\
\leq & m(\alpha) K_{G} \int_{t}^{\infty} e^{\delta(t-s)}\|C(s) v(s)-C(s) u(s)\| \mathrm{d} s \\
\leq & \omega k_{1} m(\alpha) K_{G} \int_{t}^{\infty} e^{\delta(t-s)}\|v(s)-u(s)\|_{\alpha} \mathrm{d} s \\
\leq & K_{G} m(\alpha) \omega k_{1}\|v-u\|_{\infty, \alpha} \int_{t}^{+\infty} e^{\delta(t-s)} \mathrm{d} s \\
\leq & K_{G} \delta^{-1} \omega k_{1} m(\alpha)\|v-u\|_{\infty, \alpha} .
\end{aligned}
$$

Combining previous approximations it follows that

$$
\|\mathbb{M} v-\mathbb{M} u\|_{\infty, \alpha} \leq \kappa\|v-u\|_{\infty, \alpha} .
$$

Then $\mathbb{M}$ is a contraction map on $P A P\left(\mathbb{R}, \mathbb{X}_{\alpha}, \mu\right)$. Therefore, $\mathbb{M}$ has unique fixed point in $P A P\left(\mathbb{R}, \mathbb{X}_{\alpha}, \mu\right)$, that is, there exist unique $u \in P A P\left(\mathbb{R}, \mathbb{X}_{\alpha}, \mu\right)$ such that $\mathbb{M} u=u$. Therefore, Eq. (1.2) has unique doubly-weighted pseudo-almost periodic mild solution.

\section{Application}

To illustrate the result in Theorem 4.1, we consider the following equation.

$$
\begin{aligned}
& \frac{\mathrm{d}}{\mathrm{d} t}[v(t, \cdot)+f(t, k(t, x) \nabla v(t, \cdot))] \\
= & \frac{\mathrm{d}^{2}}{\mathrm{~d} x^{2}} v(t, \cdot)+v(t, \cdot)[\sin (a t)+\sin (b t)-2]+g(t, k(t, x) \nabla v(t, \cdot)), \quad \text { on } \mathbb{R} \times[0,1],
\end{aligned}
$$

with boundary conditions

$$
v(t, 0)=v(t, 1)=0, \quad t \in \mathbb{R},
$$

where the coefficients $a, b \in \mathbb{R}$ with $\frac{a}{b} \notin \mathbb{Q}, f: \mathbb{R} \times \mathbb{X} \mapsto \mathbb{X}_{\beta}, g: \mathbb{R} \times \mathbb{X} \mapsto \mathbb{X}$ and $k_{1}, k_{2}: \mathbb{R} \times[0,1] \mapsto \mathbb{R}$ are continuous functions. 
In order to rewrite Eq. (5.1) in the abstract form equation (1.2). We choose the spaces $\mathbb{X}=L^{2}([0,1])$, endowed with the norm $\|\cdot\|_{2}$. We also consider the operator $A: D(A) \subset$ $\mathbb{X} \longrightarrow \mathbb{X}$, given by

$$
D(A)=H^{2}(0,1) \cap H_{0}^{1}(0,1) \quad \text { and } \quad A u=u^{\prime \prime} \quad \text { for } u \in D(A) .
$$

It is well known that $A$ is the infinitesimal generator of an exponentially stable $C_{0}$-semigroup $(T(t))_{t \geq 0}$ such that $\|T(t)\| \leq e^{-\pi^{2} t}$ for $t \geq 0$. Define next a set of linear operator $A(t)$ as follows:

$$
\left\{\begin{array}{l}
D(A(t))=D(A)=H^{2}([0,1]) \cap H_{0}^{1}([0,1]), \\
A(t) v=(A+\sin (a t)+\sin (b t)-2) v, \quad v \in D(A) .
\end{array}\right.
$$

Hence $D(A(t))=D(A)$ and

$$
\|A(t)-A(s)\|=\|(\sin (a t)-\sin (a s)+\sin (b t)-\sin (b s))\| \leq(|a|+|b|)|t-s|,
$$

for all $s, t \in \mathbb{R}$, then the assumption (H0) is satisfied.

$A(t)$ generate an evolution family $U(t, s)_{t \geq s}$ such that

$$
U(t, s) v=T(t-s) \exp \left(\int_{s}^{t}(\sin (a \tau)+\sin (b \tau)-2) \mathrm{d} \tau\right) v
$$

Since $\|U(t, s)\| \leq e^{-\pi^{2}(t-s)}$ for $t \geq s$ and $t, s \in \mathbb{R}$, it is easy to verify that $A(t)$ satisfy the assumption (H1) with $M=1, \delta=\pi^{2}$. Next, since $t \mapsto \sin (a t)+\sin (b t)$ is almost periodic, then $R(\omega, A(\cdot)) \in A P(\mathbb{R}, \mathcal{L}(X))$ and the assumption (H2) is satisfied. Take $\frac{1}{2}<\alpha<1$ and let $\beta, \mu$ such that $0 \leq \mu<\alpha<\beta<1$ with $2 \alpha>\mu+1$. We introduce the linear operators $B(t), C(t): \mathbb{X}_{\alpha} \mapsto \mathbb{X}$ for all $t \in \mathbb{R}$ defined by

$$
B(t) \varphi=k_{1}(t, \cdot) \nabla \varphi \quad \text { and } \quad C(t) \varphi=k_{2}(t, \cdot) \nabla \varphi \quad \text { for } t \in \mathbb{R} \quad \text { and } \varphi \in \mathbb{X}_{\alpha},
$$

where $\mathbb{X}_{\alpha}=\left(L^{2}([0,1]), H^{2}([0,1]) \cap H_{0}^{1}([0,1])\right)_{\alpha, \infty}$ and $k(t, \cdot):(0,1) \mapsto \mathbb{R}$ is continuous and almost periodic in $t \in \mathbb{R}$ uniformly in $x \in[0,1]$. Let $F: \mathbb{R} \times \mathbb{X} \mapsto \mathbb{X}_{\beta}$ be the mapping defined by

$$
F(t, \varphi)(x)=f(t, \varphi(x)), \quad \text { for } x \in[0,1]
$$

and $G: \mathbb{R} \times \mathbb{X} \mapsto \mathbb{X}$ defined by

$$
G(t, \varphi)(x)=g(t, \varphi(x)), \quad \text { for } x \in[0,1] .
$$

Let $y: \mathbb{R} \rightarrow \mathbb{X}_{\alpha}$ be the function defined by $y(t)=v(t, \cdot)$, for $t \in \mathbb{R}$. Then the system (5.1)-(5.2) takes the abstract form

$$
\frac{\mathrm{d}}{\mathrm{d} t}[y(t)+F(t, B(t) y(t))]=A(t) y(t)+G(t, C(t) y(t)), \quad t \in \mathbb{R} .
$$


Let

$$
\mu(t)=v(t)=\left\{\begin{aligned}
e^{t} & \text { if } t \leq 0 \\
1 & \text { if } t>0
\end{aligned}\right.
$$

If we assume that $f$ and $g$ are doubly-weighted $(\mu, v)$-pseudo almost periodic in $t \in \mathbb{R}$ uniformly in $u \in \mathbb{X}$ and satisfying globally Lipschitz with respect to the second argument in the following sense: there exists $K_{f}>0$ and $K_{g}>0$ such that

$$
\begin{aligned}
& \|f(t, u)-f(t, v)\|_{\beta} \leq K_{f}\|u-v\|_{L^{2}([0,1])^{\prime}} \quad \text { for all } t \in \mathbb{R} \text { and } u, v \in \mathbb{X}_{\beta}, \\
& \|g(t, u)-g(t, v)\| \leq K_{g}\|u-v\|_{L^{2}([0,1])^{\prime}} \quad \text { for all } t \in \mathbb{R} \text { and } u, v \in \mathbb{X} .
\end{aligned}
$$

Hence all assumption of Theorem (4.1) are satisfied and the equation (5.1) has a unique doubly-weighted $(\mu, v)$-pseudo almost periodic solution.

\section{Acknowledgement}

I would like to thank Prof. Habib Ouerdiane from the Department of Mathematics, Faculty of Sciences of Tunis for suggesting the subject of the paper. I would like to thank also the editor in-chief for handling the paper and the anonymous reviewer(s) for the strong comments that improved the paper.

\section{References}

[1] Diagana T., Existence of doubly-weighted pseudo-almost periodic solutions to some classes of nonautonomous differential equations. Electronic Journal of Differential Equations, 28 (2011), $1-15$.

[2] Agarwal R. P., Andrade B. de and Cuevas C., Weighted pseudo-almost periodic solutions of a class of semilinear fractional differential equations. Nonlinear Analysis, (RWA), 11 (5) (2010), 3532-3554.

[3] Blot J., Mophou G. M., N'Guérékata G. M. and Pennequin D., Weighted pseudo-almost automorphic functions and applications to abstract differential equations. Nonlinear Analysis, 71 (3-4) (2009), 903-909.

[4] Boukli-Hacenea N., Ezzinbi K., Weighted pseudo-almost periodic solutions for some partial functional differential equations. Nonlinear Analysis, 71 (9) (2009), 3612-3621.

[5] Boukli-Hacenea N., Ezzinbi K., Weighted pseudo-almost automorphic solutions for some partial functional differential equations. Nonlinear Analysis, RWA, 12 (1) (2011), 562-570.

[6] Miao C. X., Bo Z., Besov spaces and self-similar solutions for nonlinear evolution equations. J. Partial Diff. Eqs., 19 (1) (2006), 26 C47.

[7] Diagana T., Mophou G. M., and N'Guérékata G. M., Existence of weighted pseudo-almost periodic solutions to some classes of differential equations with $S^{p}$-weighted pseudo-almost periodic coefficients. Nonlinear Analysis, 72 (1) (2010), 430-438. 
[8] Diagana T., Existence of weighted pseudo-almost periodic solutions to some classes of hyperbolic evolution equations. Journal of Mathematical Analysis and Applications, 350 (1) (2009), 18-28.

[9] Diagana T., Existence of weighted pseudo-almost periodic solutions to some nonautonomous differential equations. International Journal of Evolution Equations, 2 (4) (2008), 397-410.

[10] Diagana T., Weighted pseudo-almost periodic solutions to a neutral delay integral equation of advanced type. Nonlinear Analysis, 70 (1) (2009), 298-304.

[11] Khaled G. A., A Generalized $\left(G^{\prime} / G\right)$-Expansion Method to Find the Traveling Wave Solutions of Nonlinear Evolution Equations. J. Partial Diff. Eqs., 24 (1) (2011), 55-69.

[12] Liang J., Xiao T.-J. and Zhang J., Decomposition of weighted pseudo-almost periodic functions. Nonlinear Analysis, 73 (10) (2010), 3456-3461.

[13] Liu J. H., Song X. Q. and Zhang P. L., Weighted pseudo-almost periodic mild solutions of semilinear evolution equations with nonlocal conditions. Applied Mathematics and Computation, 215 (5) (2009), 1647-1652.

[14] Liu J. H., Song X. Q., Almost automorphic and weighted pseudo-almost automorphic solutions of semilinear evolution equations. Journal of Functional Analysis, 258 (1) (2010), 196-207.

[15] Zhang L., Xu Y., Weighted pseudo-almost periodic solutions of a class of abstract differential equations. Nonlinear Analysis, 71 (9) (2009), 3705-3714.

[16] Zhang L. L., Li H. X., Weighted pseudo-almost periodic solutions for some abstract differential equations with uniform continuity. Australian Mathematical Society, 82 (2010), 424-436.

[17] Yadong S., Guo Boling, Exponential attractor for a class of nonclassical diusion equation. J. Partial Diff. Eqs., 16 (4) (2003), 289-298.

[18] Acquistapace P., Flandoli F., Terreni B., Initial boundary value problems and optimal control for nonautonomous parabolic systems. SIAM Journal on Control and Optimization, 29 (1991), 89-118.

[19] Acquistapace P., Terreni B., A unified approach to abstract linear nonautonomous parabolic equations. Rendiconti del Seminario Matematico della Università di Padova, 78 (1987), 47-107.

[20] Pazy A., Semigroups of Linear Operators and Applications to Partial Differential Equations. Applied Mathematical Sciences, 44. Springer-Verlag, New York, 1983.

[21] Amann H., Linear and Quasilinear Parabolic Problems, Birkhäuser, Berlin, 1995.

[22] Engel K. J., Nagel R., One Parameter Semigroups for Linear Evolution Equations. Graduate texts in Mathematics, Springer Verlag, 1999.

[23] Lunardi A., Analytic Semigroups and Optimal Regularity in Parabolic Problems. PNLDE Vol. 16, Birkhäauser Verlag, Basel, 1995.

[24] Diagana T., Existence of weighted pseudo-almost periodic solutions to some classes of nonautonomous partial evolution equations. Nonlinear Analysis, 74 (2011), 600-615.

[25] Diagana T., Doubly-weighted pseudo-almost periodic functions. African Diaspora Journal of Mathematics, 12 (1) (2011), 113-120.

[26] Bohr H., Almost Periodic Functions, Chelsea Publishing Company, New York, 1947.

[27] Liu J. H., N'Guérékata G. M. and Minh N. V., Topics on Stability and Periodicity in Abstract Differential Equations. Series on Concrete and Applicable Mathematics, Vol. 6, World Scientific, 2008.

[28] Diagana T., Existence and uniqueness of pseudo-almost periodic solutions to some classes of partial evolution equations. Nonlinear Analysis, Theory Methods Appl., 66 (2) (2007), 384-395.

[29] Dads E. Ait, Ezzinbi K., and Arino O., Pseudo-almost periodic solutions for some differential equations in Banach space. Nonlinear Analysis, Theory Methods Appl., 28 (7) (1997), 1141-1155. 
[30] Dads E. Ait, Ezzinbi K., Pseudo-almost periodic solutions of some delay differential equations. J. Math. Anal. Appl., 201 (287) (1996), 840-850.

[31] Blot J., Cieutat P. and Ezzinbi K., New approach for weighted pseudo-almost periodic functions under the light of measure theory, basic results and applications. Applicable Analysis, (2011), 1-34.

[32] Diagana T., Weighted pseudo-almost periodic functions and applications. C.R.A.S., 343 (10) (2006), 643-646.

[33] Diagana T., Weighted pseudo-almost periodic solutions to some differential equations. Nonlinear Analysis, 68 (2008), 2250-2260.

[34] Levitan B. M., Zhikov V. V., Almost Periodic Functions and Differential Equations. Moscow Univ. Publ. House 1978. English Translation by Cambridge University Press, 1982.

[35] Lushan Y., Deming S., The existence and uniqueness of solutions of a class of evolution equations. J. Part. Diff. Eq., 8 (1995), 233-241.

[36] Maniar L., Schnaubelt R., Almost periodicity of inhomogeneous parabolic evolution equations. Lecture Notes in Pure and Applied Mathematics, 234, Dekker, New york, 2003, 299-318. 